\title{
Review on Application of Artificial Intelligence in Civil Engineering
}

\author{
Youqin Huang ${ }^{1}$, Jiayong $\mathrm{Li}^{1}$ and Jiyang $\mathrm{Fu}^{1}{ }^{1}$ *
}

\begin{abstract}
In last few years, big data and deep learning technologies have been successfully applied in various fields of civil engineering with the great progress of machine learning techniques. However, until now, there has been no comprehensive review on its applications in civil engineering. To fill this gap, this paper reviews the application and development of artificial intelligence in civil engineering in recent years, including intelligent algorithms, big data and deep learning. Through the work of this paper, the research direction and difficulties of artificial intelligence in civil engineering for the past few years can be known. It is shown that the studies of artificial intelligence in civil engineering mainly focus on structural maintenance and management, and the design optimization.
\end{abstract}

Keywords: Artificial intelligence, civil engineering, intelligent algorithms, big data, deep learning, structural maintenance.

\section{Introduction}

The conference held at Dartmouth university in 1956 was regarded as the initial appearance of artificial intelligence (AI), which is a comprehensive discipline involving computer, control, linguistics, neuroscience, psychology and philosophy [Cao (2010)]. In the broadest sense, AI refers to the ability of a machine or artifact to perform the same functions as a human mind. According to Barr et al. [Barr and Feigenbaum (1981)], AI is the part of computer science involved in the design of intelligent computer systems, i.e., systems that exhibit the characteristics related to intelligence in human behavior, such as understanding, language, learning, reasoning, solving problems and so on [Kalogirou (2003)]. AI is widely accepted as a technology that offers an alternative way to solve complex and ill-defined problems. They can learn from examples, have strong fault tolerance which mean that they can deal with both noisy and incomplete data, as well as be able to handle non-linear problems [Kalogirou (2003)]. AI has been applied in engineering, economy, medicine, military, marine and other sectors. They have also been used for modeling, identification, optimization, prediction and control of complex systems [Mellit and Kalogirou (2008)].

\footnotetext{
${ }^{1}$ Joint Research Center for Engineering Structure Disaster Prevention and Control, Guangzhou University, Guangzhou, 510006, China.

${ }^{*}$ Corresponding Author: Jiyang Fu. Email: jiyangfu@gzhu.edu.cn.
} 
The development of AI techniques can be divided into the following five periods: the incubation period (before 1956), the formation period (1956-1970), the dark period (1966-1974), the knowledge application period (1970-1988), and the integrated development period (1986-present) [Lu, Chen and Zheng (2012)]. Especially since the late 1980s, with the in-depth development of machine learning, computational intelligence, artificial neural network and other researches, artificial intelligence technology has taken on increasingly wide utilization into other scientific fields. With the development of cloud computing and the Internet, data shows explosive growth. Different types of data are generated at different stages of the life cycle, and the application of big data technology can effectively manage these data [Motawa (2017)]. Recently, with significant advances in data acquisition and computing hardware, the field of artificial intelligence has proposed a novel machine learning technology called deep learning (DL). The key advantage of deep learning is that the multilevel features are not designed by human engineers, but by a common learning process [Huang, Li and Zhang (2018)]. This article will introduce the origins of various AI techniques and the latest developments and applications in various aspects of civil engineering.

\section{Artificial intelligent algorithms and its applications in civil engineering \\ 2.1 Genetic algorithm}

Genetic algorithm (GA) is one of the most famous evolutionary algorithms, which was proposed by Professor Holland of the University of Michigan in 1969 and summarized by Holland et al. [Holland (1975); Jong and Dejong (1975); Goldberg (1989)]. GA is a kind of random algorithm which simulates the natural process of biological evolution [Rich and Knight (1996)], inspired by Darwin's theory of evolution. Problem states in GA are represented by chromosomes that are usually denoted by binary strings. Three operators (selection, crossover, and mutation) are used by GA to simulate the natural evolution processes [Goldberg (1989); Konar (1999)]. Therefore, GA is an optimum search technology based on natural selection and survival of the fittest.

Since structural health monitoring (SHM) is becoming increasingly attractive owing to its potential ability to detect damage, Silva et al. [Silva, Santos, Figueiredo et al. (2016)] proposed an unsupervised and nonparametric genetic algorithm for decision boundary analysis (GADBA) to support the process of structural damage detection and applied the method to the Tamar Bridge in the United Kingdom (Fig. 1).

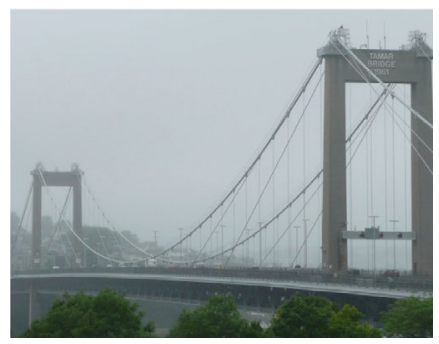

(a)

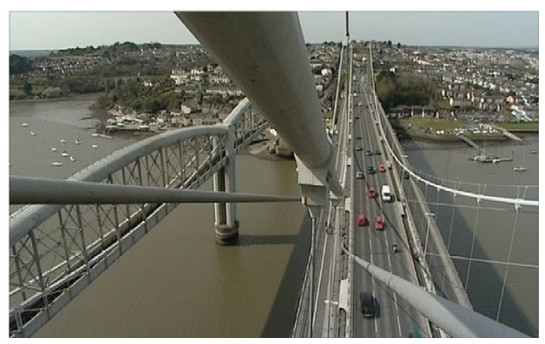

(b)

Figure 1: The Tamar Bridge viewed from (a) River Tamar margins and (b) cantilever [Silva, Santos, Figueiredo et al. (2016)] 
In view of the slow convergence rate of genetic algorithm (GA) in large-scale optimization, Mroginski et al. [Mroginski, Beneyto, Gutierrez et al. (2016)] proposed a selective genetic algorithm (SGA) for the optimization of cross-sections (solid tubes) of truss structures. SGA is based on a preliminary sensitivity analysis of each variable to reduce the search space of the evolutionary process.

Considering the cross-sectional areas of beam, column members and the types of semirigid connections as optimization variables, Truong et al. [Truong, Nguyen and Kim (2017)] adopted practical advanced analysis (PAA) and micro-genetic algorithm (GA) to optimize space steel frames with semi-rigid joints. Artar et al. [Artar and Daloglu (2018)] also employed genetic algorithm and harmony search algorithm to optimize the design of steel frames with semi-rigid connections.

Tracking the change of cable tension over time is helpful to evaluate the health condition of cable-stayed bridges, so Zarbaf et al. [Zarbaf, Norouzi, Allemang et al. (2017)] developed a method for estimating stay cable tensions of cable-stayed bridges based on genetic algorithm (GA) and particle swarm optimization (PSO), taking into account the influence of bending stiffness and vertical elongation of the cable.

Seismic base isolations are relatively perfect passive control systems, which are used to reduce the structural responses and prevent the damage of interior sensitive equipment and non-structural components. Mehrkian et al. [Mehrkian, Bahar and Chaibakhsh (2019)] proposed a multi-objective fuzzy-genetic control method for vibration reduction of structures in an irregular base-isolated benchmark building under different earthquake scenarios.

Genetic algorithm provides a general framework for solving complex system optimization, which does not depend on the specific field of the problem, so it is widely used in various fields of civil engineering. Although genetic algorithm has achieved good results in practical engineering, there are still some problems in it. Due to this, scholars all over the world have been exploring the improvement and development of genetic algorithm.

\subsection{Swarm intelligence}

The concept of swarm intelligence (SI) was first proposed in 1992 by American scholars, Hackwood et al. [Hackwood and Beni (1992)], in the molecular automata system which realizes self-organization by the interaction between adjacent individuals in the grid space. SI initially referred to algorithms inspired by the collective behavior of social insects and other animals [Bonabeau, Dorigo and Theraulaz (1999)]. Nowadays, SI refers more broadly to the research about the collective behavior of multi-component systems which coordinated by decentralized controls and self-organization [Saleem, DiCaro and Farooq (2011)]. Meta-heuristics based on swarm intelligence simulate a group of simple individuals, evolve their solutions through interactions and interactions with the environment, which show good performance on many difficult problems, and thus have become a very active research field in recent years [Lu, Chen and Zheng (2012)].

\subsubsection{Particle swarm optimization}

Particle swarm optimization (PSO) was jointly proposed by Kennedy et al. [Kennedy and Eberhart (1995)] by simulating the social behaviors of animals or insects (such as birds 
and fish). Particle swarm optimization (PSO) is a population-based global optimization technique that enables many independent solutions known as particles to move through the hyper dimensional search space to find the optimal solution. Each particle has a position vector and a velocity vector, which are adjusted in the iteration by learning the optimal local vector of the particle itself and the optimal current global vector of the whole population.

The evaluation of surrounding rock deformation in the process of underground excavation can reduce the potential damage and provide help for the reasonable selection of design parameters in the subsequent construction. However, there are many factors that affect the surrounding rock deformation of underground caverns, so it is hard to quantify their influence on the deformation of surrounding rocks. For solving this problem, Xue et al. [Xue and Xiao (2017)] presented a least squares support vector machine (LSSVM) method based on particle swarm optimization (PSO) algorithm to evaluate the deformation of surrounding rocks in underground caverns.

The squat reinforced concrete $(\mathrm{RC})$ shear wall with low aspect ratio plays an important role in resisting lateral seismic loading, so the prediction model of shear capacity of these walls is particularly essential to ensure the seismic safety of buildings. Chen et al. [Chen, $\mathrm{Fu}$, Yao et al. (2018)] proposed a model to predict the shear strength of squat RC walls based on a hybrid algorithm including the artificial neural network and particle swarm optimization algorithm (ANN-PSO).

To extract the higher modes and enhance computational efficiency during the dynamic model updating of bridge structures, Qin et al. [Qin, Zhang, Zhou et al. (2018)] applied the Kriging model and particle swarm optimization (PSO) to update the dynamic model of Jalón Viaduct in Spain (Fig. 2) using higher vibration modes under initial conditions of large vibration amplitude. As a surrogate model for a complicated finite element model, kriging model is utilized to predict analytical responses and the initial finite element model is updated by the PSO algorithm.

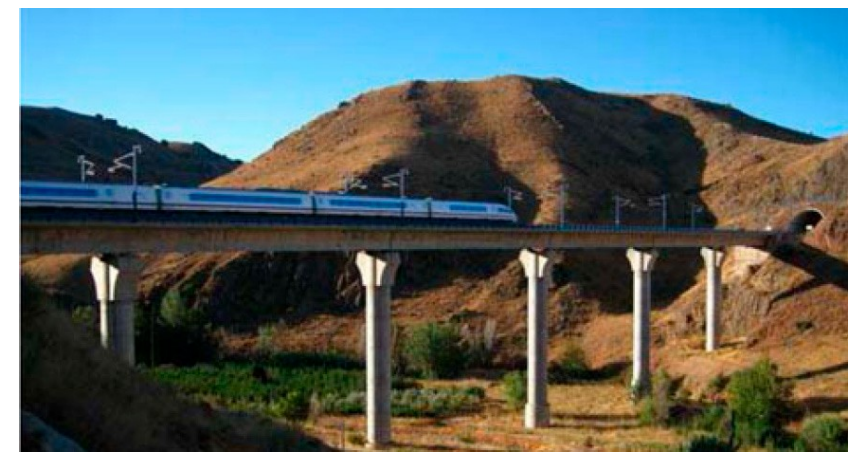

Figure 2: The Jalón Viaduct [Qin, Zhang, Zhou et al. (2018)]

Compared with genetic algorithm and ant colony algorithm, PSO algorithm has the characteristics of simple, less adjustment parameters, fast convergence, and good robustness, so it is widely used in structural design, function optimization, pattern classification, fuzzy system control and so on. 


\subsubsection{Ant colony algorithm}

Dorigo et al. [Dorigo, Maniezzo and Colorni (1991); Dorigo (1992); Colorni and Dorigo (1992)] proposed a new intelligent optimization algorithm, Ant System (AS), in the early 1990s. After several years of development, Dorigo et al. improved the Ant System into a general optimization technology, Ant Colony Optimization (ACO) [Dorigo and Caro (1999); Dorigo, Caro and Gambardella (1999)]. Its core is indirect communication between ants through pheromones, enabling them to find the shortest path from nest to food source. ACO algorithm takes advantage of the characteristics from the ant colony by designing algorithms to solve optimization problems.

Determining the noncircular critical slip surface is a key problem in slope stability analysis, which can be transformed into an optimization problem. Combined with the typical mature limit equilibrium analysis, Gao [Gao (2016)] adopted meeting ant colony optimization (MACO) to locate the critical slip surface of slopes.

To solve the problem of low computational efficiency in the traditional scheduling tool critical path method (CPM), Wang et al. [Wang, Abdul-Rahman and Chow (2016)] established a schedule acceleration model based on ant colony optimization (ACO) and $\mathrm{CPM}$ to assist construction personnel in schedule acceleration and ensure reasonable allocation of resources on critical path.

\subsubsection{Bee colony algorithm}

The artificial bee colony algorithm was proposed by Karaboga [Karaboga (2005)] in 2005. Its basic idea is that bees cooperate with each other to complete honey gathering tasks through individual division of labor and information exchange. Although individual bees have limited abilities, it is always easier for the whole colony to find high-quality honey sources without the same command. Compared with the classical optimization method, this algorithm has almost no requirements on objective function and constraint, and only takes fitness function as the basis of evolution.

Inverse analysis is a significant tool for characterizing geomechanical parameters according to the behavior of rocks under certain boundary conditions. Zhao et al. [Zhao and Yin (2016)] presented a new method for inverse analysis by taking advantage of multi-output support vector machine (MSVM) and artificial bee colony (ABC) algorithm, which was applied to recognize the parameters of the permanent shiplock slope of the Three Gorges in China. MSVM was used to plot the relationship between geomechanical parameters and displacement, and $\mathrm{ABC}$ was applied to find the optimal geomechanical parameters in the inverse analysis.

Due to the complexity of soil properties, it is difficult to establish effective response surface for traditional slope stability analysis. Kang et al. [Kang and Li (2016)] proposed an intelligent response surface method for system probabilistic stability evaluation of soil slopes, in which support vector regression (SVR) was optimized by artificial bee colony algorithm $(\mathrm{ABC})$ to establish the response surface and approximate the limit state function. 


\subsubsection{Firefly algorithm}

Yang [Yang (2008)] proposed a heuristic algorithm, the Firefly Algorithm, which was inspired by the flickering behavior of fireflies, using points in search space to simulate firefly individuals in nature. The search process is simulated as the attraction and movement process of firefly individuals. The objective function of solving the problem is measured as the position of the individual. The individual survival of the fittest process is analogous to the iterative process in which the better feasible solution replaces the worse solution in the search and optimization process [Gamdomi, Yang, Talatahari et al. (2013)].

At present, the methods used to optimize the shape and size of multi-frequency constrained truss structures are time-consuming. To improve the convergence speed and accuracy of the algorithm, Lieu et al. [Lieu, Do and Lee (2018)] proposed an adaptive hybrid evolutionary firefly algorithm (AHEFA) based on the differential evolution (DE) algorithm and the firefly algorithm (FA). Adaptive parameters are used to select the appropriate mutation scheme for the trade-off between global and local search capability.

Numerical model updating is a technique to update the experimental models of structures. However, these updating technologies still have some limitations and uncertainties, which will make the interpretation of the structures become difficult. Kubair et al. [Kubair and Mohan (2018)] established a numerical model based on firefly algorithm, which can effectively reduce the difference between experimental and analytical data in the case of frame and cantilever beam.

Although FA has a short history of development, it has some advantages over other algorithms in terms of optimization speed and accuracy. However, there are still some problems to be solved in its application, such as parameter selection, premature convergence and weak theoretical foundation.

\subsubsection{Cuckoo search}

Cuckoo search (CS) is a novel nature-based heuristic algorithm proposed by British scholars Yang et al. in 2009 on the basis of swarm intelligence technology [Yang and Deb $(2009,2010,2013)]$. The idea of the algorithm is based on the nesting parasitic behavior of cuckoos and the Lévy flight behavior of birds.

The problem of semi-active control of intelligent isolated structures under near-field and far-field earthquake excitations involves many contradictory design objectives. In order to achieve the control objective and reduce the deformation of the isolation system without significantly increasing the acceleration of the superstructure, Zamani et al. [Zamani, Tavakoli and Etedali (2017)] employed a multi-objective cuckoo search algorithm to optimize the parameters of the fractional order proportional-integralderivative (FOPID) controller.

Cuckoo search (CS) algorithm has great potential in engineering optimization because it is easy to realize and has few parameters. Rakhshani et al. [Rakhshani and Rahati (2017)] proposed an intelligent multiple search strategy algorithm (IMSS) coupled with CS and covariance matrix adaptation evolution strategy (CMAES), to effectively explore the search space and reduce the computational time to find the optimal solution. 


\subsubsection{Krill herd algorithm}

Krill herd algorithm $(\mathrm{KH})$ is a new optimization algorithm proposed by Gandomi et al. [Gandomi and Alavi (2012)] in 2012. The KH algorithm is based on the simulation of the foraging behavior by krill individuals. The objective function of krill movement is correlated with the minimum distances of each individual krill from food and from the highest population density. The position of an individual krill is determined by three main factors: (i) movement caused by other krill individuals, (ii) foraging activities, and (iii) random diffusion [Gandomi and Alavi (2012)]. KH not only has good local and global optimization performance, but also can effectively balance global search and local development to avoid falling into local extreme values [Wang, Guo, Gandomi et al. (2014); Wang, Gandomi and Alavi (2014); Guo, Wang and Gandomi (2014)].

The engineering optimization problems are usually non-linear and it is difficult to obtain the optimal solutions. In order to evaluate the application of $\mathrm{KH}$ algorithm in engineering, Gandomi et al. [Gandomi and Alavi (2016)] applied it to solve four engineering problems, including tubular column design, three-bar truss design, speed reducer design, and helical compression spring design.

\subsection{Artificial neural networks}

Artificial neural networks (ANNs) may be defined as a structure of tightly interconnected adaptive simple processing elements (named artificial neurons or nodes) which are able to perform large-scale parallel computations for data processing and knowledge representation [Schalkoff (1997); Basheer and Hajmeer (2000)]. In 1943, McCulloh et al. [McCulloch and Pitts (1943)] proposed the first artificial neuron model (MP model), which adopted simplified signal propagation mechanism to imitate some basic functions of human brain neurons, thus laying the foundation for the development of early neural computing.

Adeli et al. [Adeli and Yeh (1989)] published the first journal article on the civil/structural engineering applications of neural networks. Since then, neural network has been widely used in civil engineering. Artificial neural network (ANN) is a kind of technical reconstruction of biological neural network in a simplified sense. Its main task is to build a practical artificial neural network model according to the principle of biological neural network and the need of practical application, design corresponding learning algorithm, and simulate some intelligent activities of human brain. Finally, it is implemented technically to solve practical problems. A three-layer feedforward network [Hecht-Nielsen (1990); Basheer and Hajmeer (2000)] is shown in Fig. 3, which consists of input layers, hidden layers and output layers. The input layers contain the number of independent variables, and the output layers correspond to the solutions of the studied problems. 


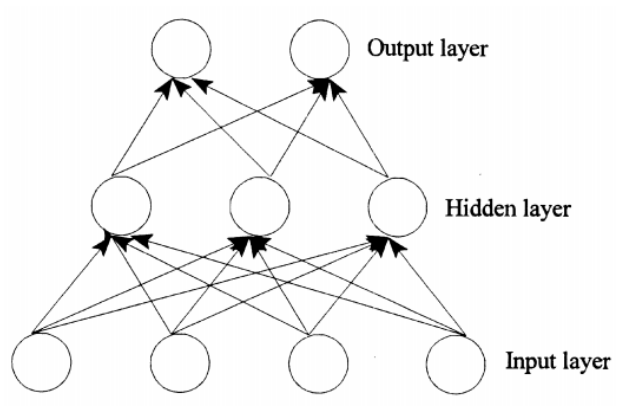

Figure 3: Three-layer feedforward network [Hecht-Nielsen (1990); Basheer and Hajmeer (2000)]

The potential surface settlement is one of the most dangerous factors in infrastructure tunnel excavations, so accurate prediction of surface maximum settlement (MSS) is the key to reduce the risk of surface failure. Hasanipanah et al. [Hasanipanah, NoorianBidgoli, Armaghani et al. (2016)] proposed an artificial neural network (ANN) hybrid model based on particle swarm optimization (PSO) to predict MSS caused by tunneling along the line 2 of Karaj subway.

In order to minimize the total cost, bridges should be designed with safety and durability. Garcia-Segura et al. [Garcia-Segura, Yepes and Frangopol (2017)] presented an integrated multi-objective harmony search with artificial neural networks (ANNs) to reduce the computational time of finite element model used in deck analysis. The optimal design of the post-tensioned concrete box girder road bridge is carried out for the purpose of the cost, the overall safety factor and the initial corrosion time.

Fiber Reinforced Polymers (FRP) are widely adopted in passive confinement to Reinforced Concrete (RC) for improving compressive strength and ductility. Casardi et al. [Cascardi, Micelli and Aiello (2017)] presented a model based on artificial neural network (ANN) for the prediction of FRP-constrained concrete compressive strength. Compared with the general models, the proposed one establishes an analytical relationship without considering traditional effectiveness parameter.

Compared with reinforced concrete products, glass fiber-reinforced polymer (GFRP) bar reinforced concrete structures have higher durability. The evaluation of the bonding properties of GFRP bars to concrete is of great significance to the design and implementation of the polymer-matrix composites (PMCs). Yan et al. [Yan, Lin, Azarmi et al. (2017)] proposed a hybrid modeling to predict the bond strength of GFRP bars to concrete by using the strong nonlinear mapping ability of artificial neural network (ANN) with the global searching ability of genetic algorithm (GA).

Improper structural design may lead to sudden collapse of multistory reinforced concrete buildings, which needs proper analysis of all factors that affects the structure. Because the traditional neural network model has poor convergence under the training of local search optimization algorithm, which cannot achieve the expected learning effect, Chatterjee et al. [Chatterjee, Sarkar, Hore et al. (2017)] proposed a model based on neural network-particle swarm optimization (NN-PSO) to predict the structural failure of a multistoried reinforced concrete building. 
The promotion of recycled aggregate concrete (RAC) can effectively reduce construction waste, and different techniques have been used to predict and evaluate the properties of RAC in recent years. Naderpour et al. [Naderpour, Rafiean and Fakaharian (2018)] applied ANN model trained by 139 sets of existing data to predict the compressive strength of RAC. The proposed ANN model has 6 input features, such as water-cement ratio, water absorption rate, fine aggregate, natural coarse aggregate, recycled coarse aggregate, and water-total material ratio.

The ANNs perform well on some tasks while poorly on others. Specifically, they are suitable for tasks involving incomplete data sets, fuzzy or incomplete information, and for highly complex and ill-defined problems where human decisions are often made intuitively [Kalogirou (2001)]. After decades of development, neural networks have been widely used in various fields of civil engineering, including structural optimization, structural condition assessment and health monitoring, structural control, structural material characterization and modeling, construction engineering, highway engineering, etc. [Adeli (2001)]. In recent years, deep learning has become a hot area of research in neural networks and made great breakthroughs in speech recognition, image recognition, natural language processing and other fields. In civil engineering, deep learning and big data have also attracted the attention of more and more researchers, which will be discussed in Section 3 and Section 4.

\section{Big data}

In the last few years, the rapid development of big data technique has become a hot topic in the scientific and technological fields, business area and even governments around the world [Walker (2014)]. Nature first launched the big data as a special issue in 2008. In February 2011, Science published a special journal Dealing with Data, mainly focusing on the theme of big data in scientific researches, demonstrating the importance of big data to academic studies. Big data [Zikopoulos and Eaton (2011); Meijer (2011)] has three defining attributes, namely (i) volume (TB, PB of data and beyond); (ii) variety (structured data and unstructured data); and (iii) velocity (continuous streams of the data). In engineering, we must adapt data from many new sources, such as GPS, wireless devices, sensors, and streaming communication generated by machine-to-machine interactions [Tran (2016)]. Data is continuous, unstructured and unconstrained by the rigid structure of rows and columns, which is difficult to deal with by traditional methods. Therefore, the application of big data is extremely urgent.

Autonomous data sources with distributed and decentralized controls are the main characteristics of big data applications. Because it is autonomous, each data source can generate and collect information without requiring (or relying on) any centralized control [Wu, Zhu, Wu et al. (2014)]. This is similar to the World Wide Web (WWW) setup, where each Web server provides a certain amount of information and each server is fully operational without relying on other servers. On the other hand, if the entire system has to rely on any centralized control unit, the sheer volume of data can also leave applications vulnerable to attacks or failures. For major big-data-related applications, such as Google and Facebook, a number of server farms are deployed around the world to ensure uninterrupted service and fast response for local markets. This source of autonomy is not 
only the solution of technological design, but also the result of different national/regional legislation and regulations.

\subsection{Structural maintenance}

For large scale bridges, monitoring of structural components is the main consideration for maintenance and operation. However, monitoring of mechanical and electrical components is also important for movable bridges. Catbas et al. [Catbas and Malekzadeh (2016)] proposed a machine learning algorithm for processing large amounts of data collected from mechanical parts of Sunrise Bridge in Florida. To monitor the condition of the mechanical parts (such as rack and pinion, Fig. 4), accelerometers were installed to monitor their vibrations. The proposed approach includes the training and monitoring phases. The training phase focused on extracting statistical characteristics, conducting cross correlation analysis (CCA) and robust regression analysis (RRA). The monitoring phase includes tracking for errors related to the derived models.

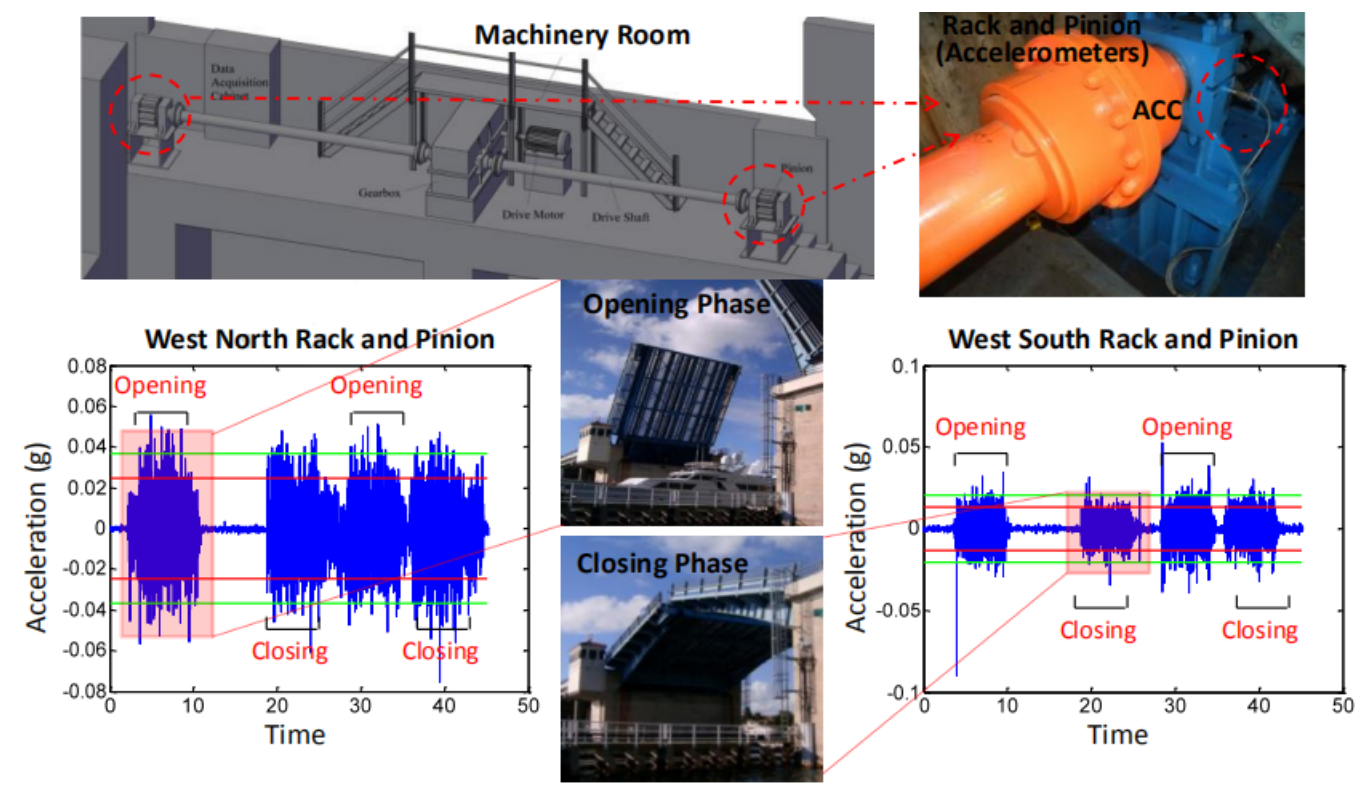

Figure 4: Sample measurement from the Rack and Pinion during opening and closing phases of The Sunrise Bridge [Catbas and Malekzadeh (2016)]

Although some work has been done on using data management infrastructure and machine learning technologies for structural monitoring, few platforms have been investigated to seamlessly integrate full spectrum input data. Liang et al. [Liang, Wu, Liu et al. (2016)] developed a multi-scale structural health monitoring (SHM) and measuring system based on the Hadoop Ecosystem (MS-SHM-Hadoop) for monitoring and evaluating the serviceability of bridges.

Existing evaluation methods using National Bridge Inventory (NBI) data focus on individual bridge components, which may not be sufficient to make full use of the accumulated data. According to NBI, Kim et al. [Kim and Queiroz (2017)] constructed a 
large data set containing 1,002,172 bridge decks and superstructures, which is used to explore the degradation trend of bridges and the corresponding model development.

The existing models applied in damage detection require expensive computing, which results in necessity of the use of parallel computing based on Multi-processor System on Chip, MPSoC, architecture. To overcome this problem, Tran [Tran (2016)] developed a new distributed parallel processing model, which is based on single instruction and multiple data (SIMD) and adopts the "master-slave" structure. SIMD technology enables us to segment big data in data sets and process on different processors and CPUs at the same time, which saves a lot of time. The effect of new model for the local damage detection in real time of the plane steel truss structures was demonstrated through numerical examples.

At present, researches on damage detection are mainly focused on the utilization of wireless sensor networks (WSN). However, the data collected from these networks have been unmanageable. To effectively manage and analyze the huge sensor data sets generated by existing WSN Settings, Rosales et al. [Rosales and Liyanapathirana (2017)] proposed a feature extraction technique which is an outlier detection technique based on time domain statistical analysis, Auto Regressive (AR) and external input method (ARX).

The support vector machine (SVM) algorithms have been widely accepted as an effective tool for feature extraction and damage detection, while it is still a big challenge to choose proper parameters. Therefore, Gui et al. [Gui, Pan, Lin et al. (2017)] explored the feasibility of combining data-driven SVM with optimization technology to enhance feature extraction and parameter optimization in structural health monitoring (SHM) and damage detection. The effectiveness and sensitivity of enhanced SVM for damage detection are studied by three optimization techniques: grid search method, particle swarm optimization and genetic algorithm.

Currently, building information modeling (BIM) systems have been developed to help store various structured data of buildings. However, these systems cannot make full use of the intelligent functions of BIM systems to capture the knowledge and unstructured data used in the operation of building systems in a usable format. Therefore, Moteva [Motawa (2017)] applied data capture technique (spoken dialogue systems, SDSs) to integrate the knowledge of building operations into the BIM system to acquire the operational knowledge, especially the maintenance and renovation of buildings.

With the rapid development of traffic construction, highway engineering quality monitoring has a new challenge. The previous management mode of "artificial + paper recording" is basically unable to achieve scientific management of engineering quality. Xiao et al. [Xiao and Zhou (2017)] conducted deeply analysis of generation, storage, processing and characteristics of big data generated by quality monitoring of highway engineering, made a detailed description of key technologies for big data processing and discussed the application of big data in quality monitoring of highway engineering.

Nowadays, highway authorities are under pressure to reduce congestion and improve environmental outcomes. Effective data management can improve the data flexibility of traffic management departments and enable them to meet the growing demands of road network. Aziz et al. [Aziz, Riaz and Arslan (2017)] proposed a platform integrating various technologies and systems of the highway management authority and its supply 
chain, allowing data to flow continuously throughout the life cycle of an asset, thus achieving seamless, collaborative and efficient work.

The dynamic characteristics of cable-stayed bridges are considered as valuable indicators to determine their performance in structural health monitoring (SHM). However, some factors, such as operational and environmental conditions, may cause high interference to the vibration response. Due to the robustness of data-intensive machine learning techniques for data classification, Pan et al. [Pan, Azimi, Yan et al. (2018)] developed a support vector machine (SVM) based data driven framework for structural diagnosis and damage detection, combined with enhanced feature extraction technology to rapidly evaluate the state of large cable-stayed Bridges (Fig. 5).

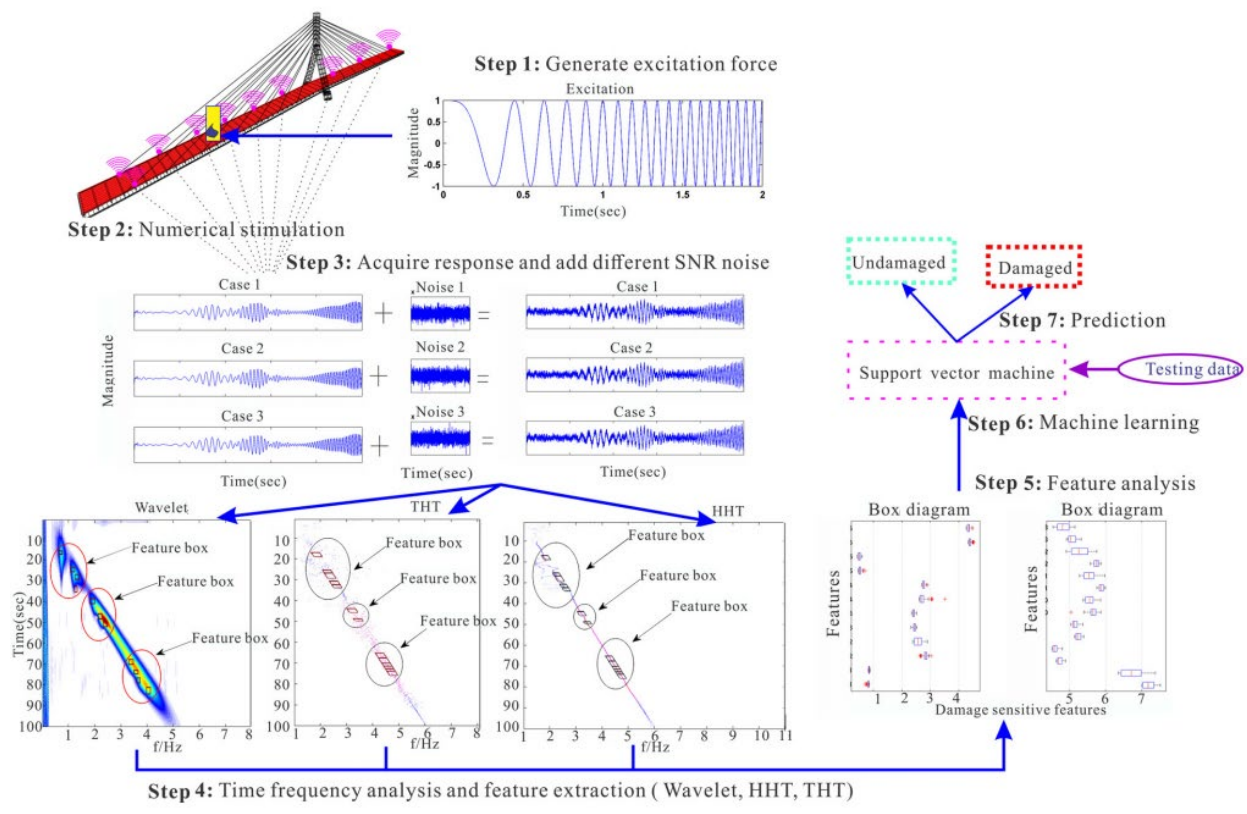

Figure 5: Framework of data-driven data mining process for SHM and damage detection [Pan, Azimi, Yan et al. (2018)]

Though smart-phone accelerometers are imperfect sensors, they can provide valuable information to SHM. Matarazzo et al. [Matarazzo, Santi, Pakzad et al. (2018)] developed an application for Harvard Bridge, showing that acceleration data collected using smartphones in mobile vehicles contained consistent and important indicators of the first three modal frequencies of the bridge. This result enables local governments to collect bridge vibration data at low cost, thus contributing to more effective management and informed decision-making.

As the increasing amount of data generated over the project lifecycle, data modeling, visualization and simulation have become main aspects of the decision to support design, build, operate, and maintain build assets. Delgado et al. [Delgado, Butler, Brilakis et al. (2018)] proposed a method of building information modeling (BIM) using structural monitoring data dynamically, which can automatically generate the parameter BIM 
models of the structural monitoring systems including time series sensor data, and realize data-driven and dynamic visualization in the interactive 3D environment.

It is necessary to identify the sources of uncertainty and quantify their impact on diagnosis and prognosis, which is conducive to effective risk management. Cai et al. [Cai and Mahadevan (2018)] developed a framework to apply big data analytics to the calculation and uncertainty quantification of structural diagnosis and prognosis. In the study, image processing of acquired data and subsequent diagnosis using Bayesian updating were parallelized via MapReduce by converting the inputs and outputs into keyvalue pairs.

A large amount of pavement condition data has great potential to support data-driven pavement management decisions, such as the choice of materials and time in pavement maintenance. However, most agencies face a variety of technical and data integration problems that lead to inefficient use of the collected data. Abdelaty et al. [Abdelaty, Jeong and Smadi (2018)] analyzed the technical challenges and data integration obstacles of pavement maintenance, and a set of suggestion was put forward to help highway management agencies make full use of pavement condition data for the implementation of road asset management.

At present, the main feature of the surface defect detection technologies applied to railway track is the identification of the defect presence, but the method to find more details of defects, including their boundaries, is rarely discussed. Zhuang et al. [Zhuang, Wang, Zhang et al. (2018)] proposed a double-layer data-driven framework for visual automatic inspection of rail surface cracks. Based on images of rails, the proposed framework can firstly detect the location of cracks, and then automatically obtain the boundary of cracks through feature-based linear iterative crack aggregation.

In rail infrastructures, condition-based monitoring is used to assess the actual health conditions of assets for effective maintenance. Jamshidi et al. [Jamshidi, Hahizadeh, Su et al. (2018)] developed a decision support method based on expert systems for conditionbased maintenance of rails. The proposed approach makes use of axle box acceleration signals and rail video images of a track in the Dutch railway network, which provides a huge amount of data.

\subsection{Design optimization and energy management}

In practice, the combination of complex problem formulation and long simulation times means that optimization is rarely used in the design of buildings and civil structures. Using the surrogate models to quickly evaluate design performance can lead to more indepth exploration of design space and reduce the computational time of the optimization algorithms. Tseranidis et al. [Tseranidis, Brown and Mueller (2016)] explored the application of data-driven approximation algorithms commonly known as surrogate modeling in the early design of structures.

Due to the situation that most problems of geotechnical engineering are highly nonlinear and multivariable, it takes a lot of calculation cost to carry out complex numerical analysis such as finite element simulations. In order to reduce costs, Zhang et al. [Zhang, Goh and Zhang (2016)] explored multivariate adaptive regression spline (MARS), a surrogate model 
technique for analyzing multivariate problems, to approximate the relationship between input and output with big data.

At present, lots of researchers have investigated the energy management systems from the macro perspective of utilities and power companies, and few studies have put forward dynamic operating strategies for effectively saving energy costs in household appliances. Therefore, Chou et al. [Chou and Ngo (2016)] presented a real-time smart grid data analysis framework for the high-efficiency energy saving system at appliance level for residential buildings, which integrates data analytics and dynamic multi-objective optimization models to generate energy consumption patterns and alternative energy saving solutions at the device level.

Although physical methods aiming at calculating building energy consumption behavior are accurate, these methods are impractical in some applications due to the necessity of continuously inspecting and gathering data for all input parameters. Chokor et al. [Chokor and ELAsmar (2017)] proposed a new Leadership in Energy and Environmental Design (LEED) rating system performance evaluation method based on the study of customized prediction model driven by building energy consumption data. The research results show that the gradient propulsion regression model is superior to other regression models in predicting and studying building energy consumption, which is helpful for practitioners to make better choices throughout the project life cycle.

\subsection{Other applications}

For complex civil engineering systems, Gandomi et al. [Gandomi, Sajedi, Kiani et al. (2016)] presented a multi-objective genetic programming algorithm (MOGP) for Big Data mining. A big data processing scheme based on batch processing and parallel computing of distributed computers was established for modeling the total timedependent creep of concrete, which prove the proposed method is an effective tool for non-linear engineering problems.

Construction waste analysis increasingly relies on high-performance computation and large-scale data storage. Using traditional techniques to store these data sets and process the data in real time for complex analysis is a challenging task. Bilal et al. [Bila, Oyedele, Akinade et al. (2016)] proposed a big data architecture for construction waste analysis (Fig. 6) to manage and analyze these unprecedented data. 


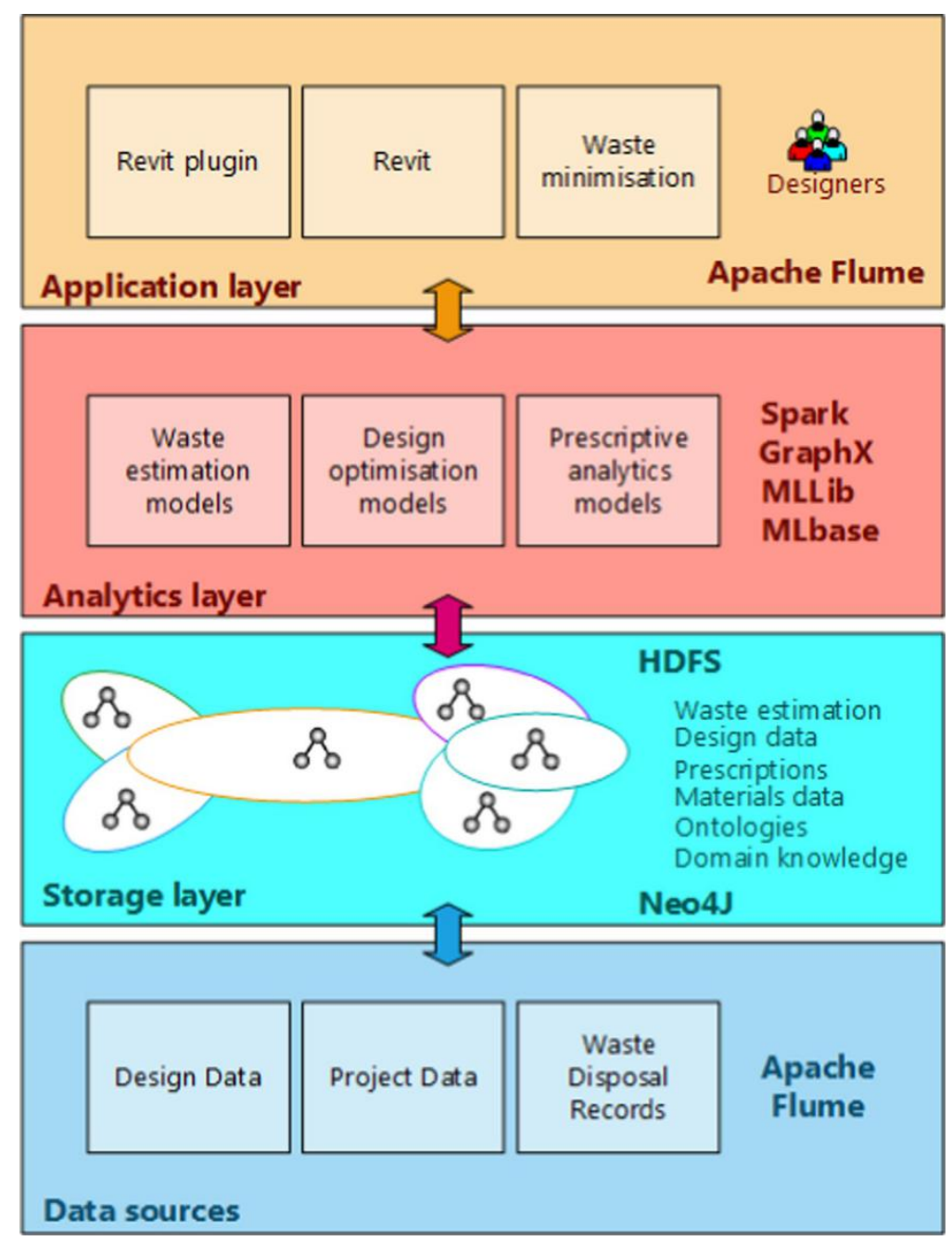

Figure 6: Big Data architecture for construction waste analytics [Bila, Oyedele, Akinade et al. (2016)]

Hundreds of images and video are taken during the construction process, but most of them quickly become obsolete without proper localization planning documents and time. Han et al. [Han and Golparva-Fard (2017)] discussed the potential of using big visual data together with BIM for construction performance monitoring to quickly annotate and report on the construction of various applications, including communication about the progress, quality and safety of problems.

Although the structures always produce a huge amount of data in their whole life cycles in the field of civil engineering, the application of big data technique in the civil engineering field lags behind other fields. With the development of technologies needed for storing, computing, processing, analyzing and visualizing big data, more and more scholars have applied such technologies in civil engineering. By reviewing the application of big data technique in various aspects of civil engineering, it can be 
concluded that the development of big data technologies in structural maintenance is relatively fast, while its developments in other aspects are slow.

\section{Deep learning}

Since the late 1980s, the development of machine learning has gone through two stages: shallow learning and deep learning. The difference between shallow and deep learners lies in the depth of their credit assignment paths, which are chains of possibly learnable, causal links between actions and effects [Schmidhuber (2015)]. In 2006, Hinton et al. [Hinton and Salakhutdinov (2006)] presented a paper in Science, which attracted the attention of deep learning in academia and industry. Deep learning is a class of machine learning algorithm that applies multiple layers to progressively extract higher-level features starting with the raw input [Deng and Yu (2014)]. In recent years, various deep learning algorithms, such as deep neural network, convolutional neural network and deep belief network, have been successfully applied in computer vision, speech recognition, natural language processing [LeCun and Bengio (2015)], and their applications in civil engineering are mainly in vision-based structural health monitoring. The outstanding advantage of deep learning is that it can model high-level abstractions in data by using architectures composed of multiple nonlinear learning layers. Each layer corresponds to different levels of nonlinear abstraction, so that the deep network has a higher characterization ability [Gan, Wang and Zhu (2016)].

With the development of deep models, the question of "why deep neural network is needed instead of shallow neural network method" is gradually studied. Traditional shallow neural networks are unable to extract high-level features, and even need further post-processing to explain the extracted high-dimensional features, while deep learning methods can use more training data and more complex network architecture to solve these problems [Xu, Wei, Bao et al. (2019)].

\subsection{Structural health monitoring}

Pixel-level accuracy is crucial for automation of pavement crack detection, but the current Convolutional Neural Network (CNN)-based methods have limitations in respect of pixel-level accuracy. Zhang et al. [Zhang, Wang, Li et al. (2017)] proposed a CNNbased architecture, named as CrackNet (Fig. 7), for automatic detection on cracks of three-dimensional asphalt pavement (Fig. 8). CrackNet composes of five layers and includes more than one million parameters trained in the learning process to ensure pixellevel accuracy. In order to improve learning ability and performance, Zhang et al. [Zhang, Wang, Fei et al. (2018)] proposed an improved architecture of CrackNet, called CrackNet II, which has a deeper architecture with more hidden layers but fewer parameters. 

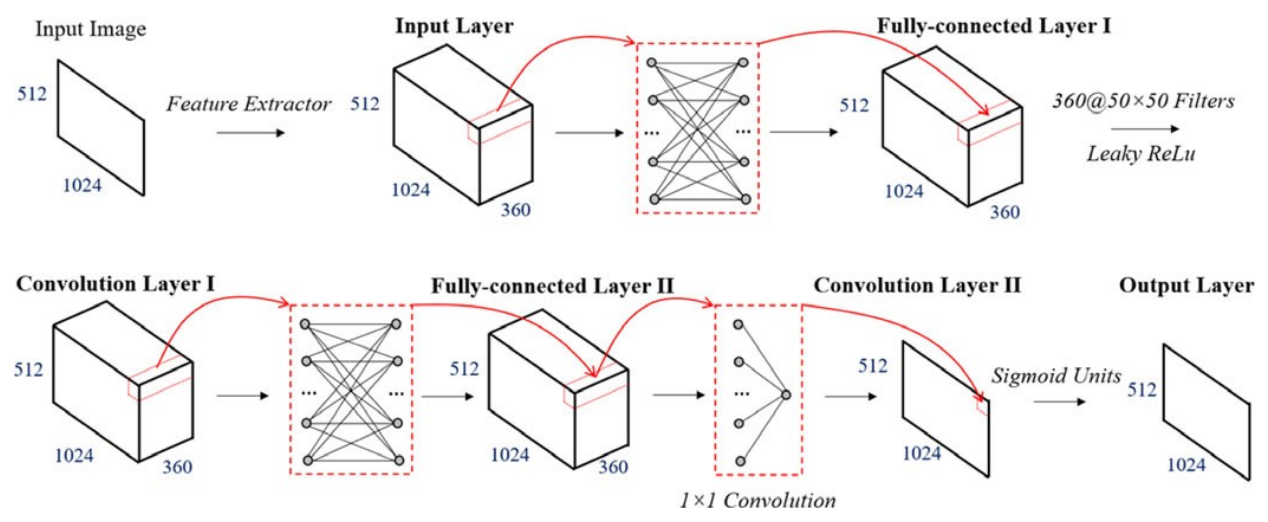

Figure 7: Architecture of CrackNet [Zhang, Wang, Li et al. (2017)]

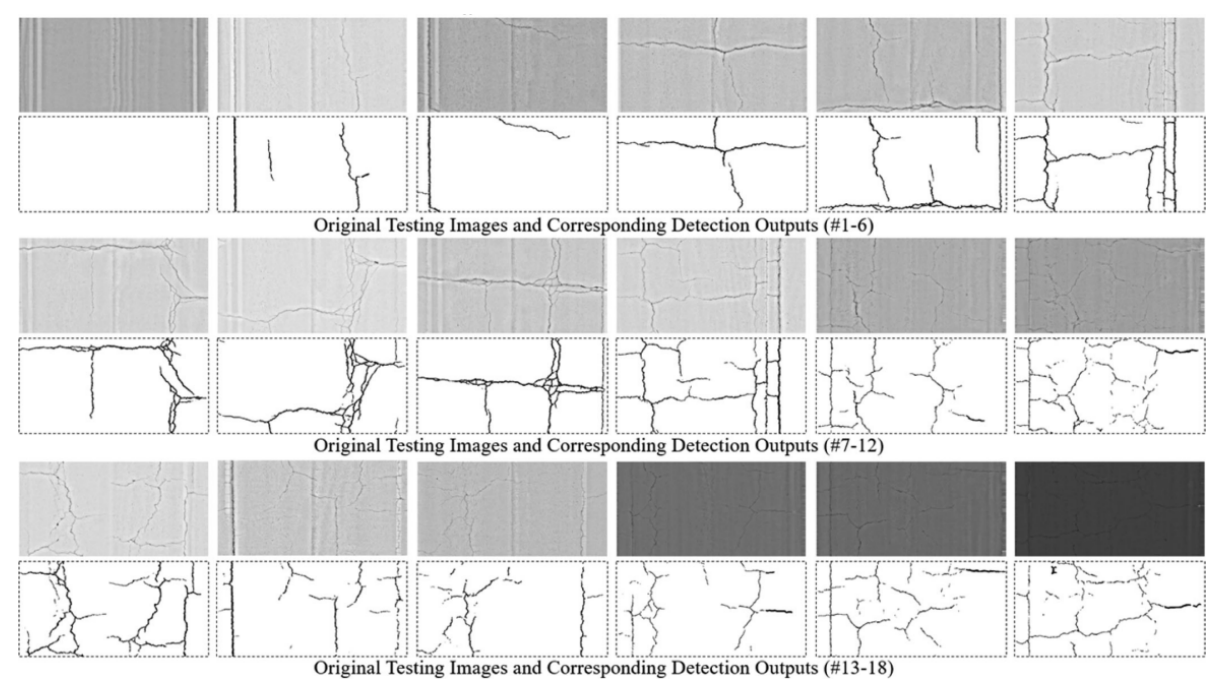

Figure 8: Typical testing images correctly classified by CrackNet [Zhang, Wang, Li et al. (2017)]

Crack and leakage defects are commonly observed on the surface of shield tunnel lining which affects the safety of tunnel structure, while the DL techniques of CNNs are unable to locate defect boundaries effectively. Based on deep learning using hierarchies of features extracted by the fully convolutional network (FCN), Huang et al. [Huang, Li and Zhang (2018)] presented an image recognition algorithm to conduct semantic segmentation of cracks and leakage defects of metro shield tunnel.

Effective condition monitoring strategies can help engineers to carry out reasonable pavement maintenance, so as to reduce maintenance costs. However, existing crack detection methods still have limitations due to their inability to overcome the inherent challenges associated with these pavement images, such as inheterogeneity of cracks and diversity of surface textures. Gopalakrishnan et al. [Gopalakrishnan, Khaitan and Choudhary (2017)] developed a vision-based pavement crack detection system, which uses a pre-trained deep learning model to detect cracks in pavement images by means of transfer learning and regional adaptive methods. 
The automatic pavement crack detection systems are faced with two challenging problems: (1) due to complicated pavement textures and intensity inhomogeneity, the noise cannot be effectively removed; (2) sealed cracks and cracks with similar intensity and width cannot be separated correctly. Zhang et al. [Zhang, Cheng and Zhang (2018)] proposed a unified crack and sealed crack detection method, which can simultaneously detect and separate cracks and sealed cracks under the same framework. The trained deep convolutional neural network is used to pre-classify the crack, sealed crack, and background regions of pavement images.

The crack of steel box girders will reduce the reliability of the structure and shorten the service life of the bridge. Because the complicated background information of the actual bridge structure often appears in the image, which may lead to the error of the crack identification, an advanced algorithm is need to solve this problem. $\mathrm{Xu}$ et al. [ $\mathrm{Xu}, \mathrm{Li}$, Zhang et al. (2018)] proposed an identification framework based on a restricted Boltzmann machine (RBM), a deep learning technique, for the identification and extraction of cracks from images with a complex background in a steel box girder of bridges.

Nowadays, it is still a challenging problem to extract damage-sensitive and noise-robust features from structural response. To automatically extract features from low-level sensor data, Lin et al. [Lin, Nie and Ma (2017)] presented a method for structural damage detection based on a deep convolutional neural network (CNN) with customized architecture, which can locate accuracy on both noise-free and noisy data set.

Due to the severe environment of civil structures, the measured data of the structural health monitoring systems are subject to multiple interference, which seriously affect the data analysis results. Existing data cleaning methods mainly focus on noise filtering, while the detection of incorrect data requires professional knowledge and is timeconsuming. In order to automatically detect multi-pattern anomalies of data, Bao et al. [Bao, Tang, Li et al. (2019)] proposed a data anomaly detection method based on computer vision and deep learning. The framework of this method includes two steps: data transformation through data visualization, and the construction and training of a deep neural network for anomaly classification.

In structural health monitoring, image processing techniques (IPTs) are mainly used to process images to extract defect features, such as cracks on concrete and steel surfaces. However, the varying real-world situations (e.g., changes in light and shadow) can affect the use of IPTs. In order to overcome these challenges, Cha et al. [Cha, Choi and Buyukozturk (2017)] proposed a visual-based method using a deep architecture of convolutional neural networks (CNNs) (Fig. 9) to detect concrete cracks without calculating defect characteristics, which is less affected by the noise caused by lighting, shadow casting, blur, and so on. 


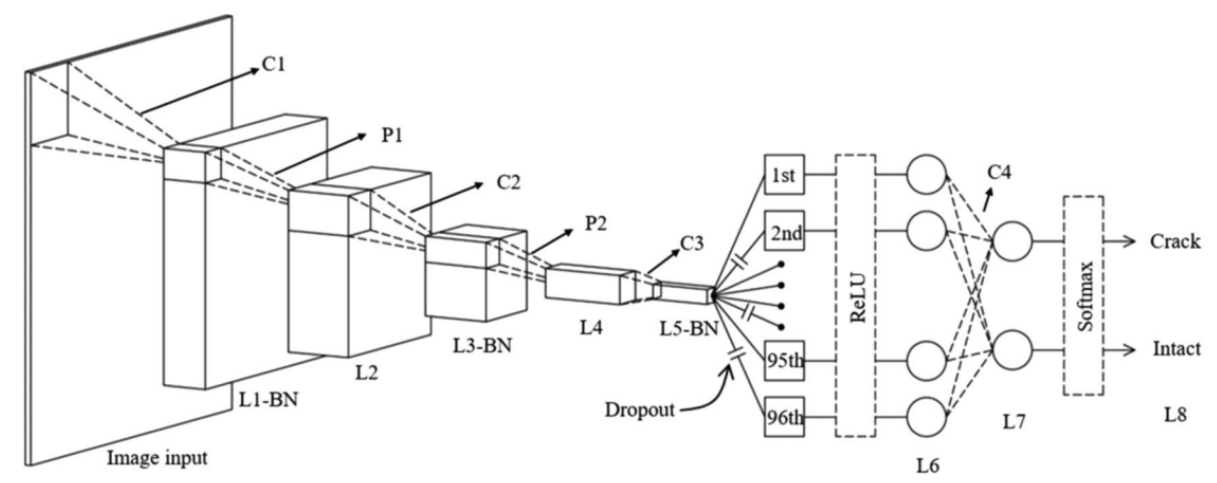

Figure 9: Overall architecture: L\#: layers corresponding to operations (L1, L3, L5, and L7: convolution layers; L2 and L4: pooling layers; L6: ReLU layer; L8: softmax layer); C\#: convolution; P\#: pooling; BN: batch normalization [Cha, Choi and Buyukozturk (2017)]

Currently, deep learning-based cracks detection methods cannot automatically extract cracks rapidly and accurately at the pixel level. Ni et al. [Ni, Zhang and Chen (2019)] proposed a framework based on convolutional neural network, which realizes task automation through convolutional feature fusion and pixel-level classification. Based on the concrete crack image database, the fracture description network with an empirically optimal fusion strategy, termed the crack delineation network, is trained and tested.

Most of researches on crack detection are based on image classification and/or object detection using boundary box, which cannot provide accurate information of crack path and density. To distinguish "cracked" and "non-cracked" pixels, Dung et al. [Dung and Anh (2019)] proposed a crack detection method based on the deep full convolutional network (FCN) for semantic segmentation of concrete crack images. The encoderdecoder FCN is trained end-to-end to segment an image of concrete crack into "crack" and "non-crack" pixels, for the task of crack detection and crack density evaluation.

Health monitoring of large and complex structures is considered as a significant technology in structural engineering research, where research has been reported mostly on vibration-based health monitoring. Based on integration of synchrosqueezed wavelet transform (SWT), an unsupervised Restricted Boltzmann Machine (RBM), and neural dynamics classification (NDC) algorithm, Rafiei et al. [Rafiei and Adeli (2018a)] proposed a new unsupervised learning model for the global and local condition assessment of structure by using the vibration response data.

After construction is completed, inspectors are required to evaluate cracks and finishing defects in building components, but sampling methods may leave out some defective areas. Liu et al. [Liu and Yan (2017)] developed a new damage detection method, named as transfer learning on convolutional activation features (TLCAFs), for the detection of cracks, hollow and finishing defects. It is based on an active transfer learning network, where deep learning networks are used to detect and verify visual defects.

Most computer vision-based approaches for structural damage detection require pre- and postprocessing techniques, which are time-consuming and can detect only one type of damage. To provide near-real-time simultaneous detection of multiple types of damage, Cha et al. [Cha, Choi, Suh et al. (2018)] developed a Faster Region-based Convolutional 
Neural Network (Faster R-CNN)-based structural visual inspection method (Fig. 10) to detect five types of surface damages: concrete cracks, steel corrosion (medium and high levels), bolt corrosion, and steel delamination.

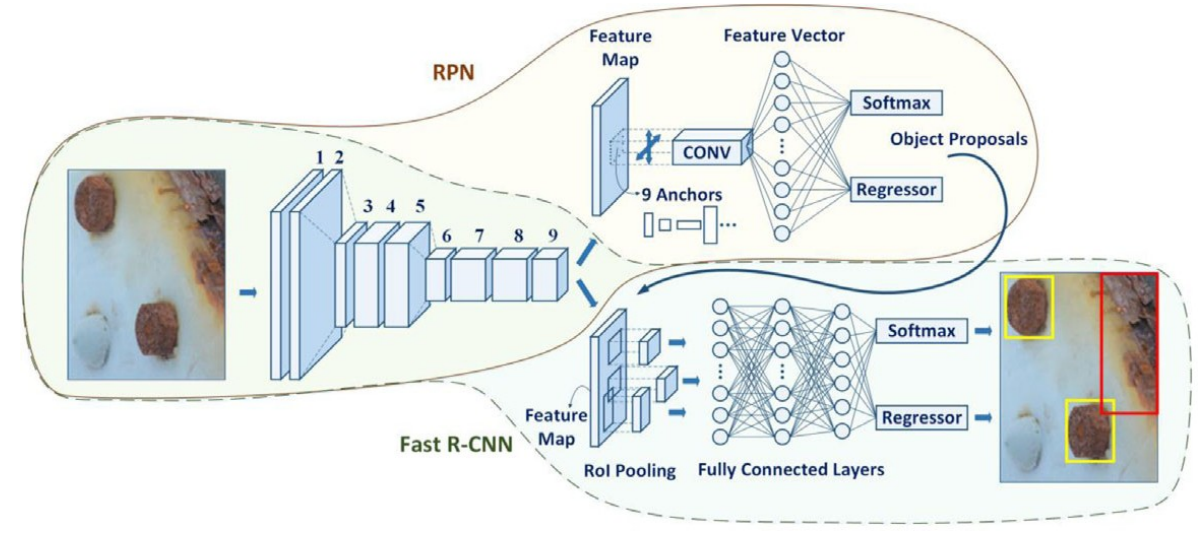

Figure 10: Schematic architecture of the Faster R-CNN [Cha, Choi, Suh et al. (2018)]

In some engineering areas, machine learning has been a great success in recent years, but it relies heavily on large amounts of data, especially labeled ones. Transfer learning is a very effective tool to reduce the dependence of machine learning precision on available data size and to maximize the use of existing data. Gao et al. [Gao and Mosalam (2018)] proposed the Structure ImageNet, inspired by deep learning, transfer learning and imageNet, for vision-based damage identification of structural components.

Vibration-based methods are susceptible to uncertainties in the process of damage identification. Pathirage et al. [Pathirage, Li, Li et al. (2018)] proposed an autoencoder based deep learning framework for structural damage identification, which can be used to obtain optimal solutions for pattern recognition problems of highly non-linear nature, such as learning the mapping between vibration characteristics and structural damage.

Vision-based autonomous detection of concrete surface defects is significant for efficient maintenance of infrastructures. However, most current vision-based inspection methods can only perform one specific task, which is either defects detection or localization. To address these limitations, Li et al. [Li, Yuan, Zhang et al. (2018)] proposed a unified and purely vision-based method for defects detection and localization network, which can detect and classify typical defect types under various conditions and simultaneously localize defects geographically without the need of external localization sensors. Ren et al. [Ren, Chen, Li et al. (2018)] developed a DL-based computational algorithm to identify the damage load condition through learning the residual plastic deformation of structure under the load, and the method is further applied in inversely predicting the the impact load on shell structures [Chen, Li, Chen et al. (2019)].

Except the damage detection, the fatigue reliability assessment is also paid attention to for engineering structures. Lu et al. [ $\mathrm{Lu}$, Noori and Liu (2017)] proposed a machine learning method to evaluate the fatigue reliability of welded steel bridge decks under stochastic truck loads, where a learning machine integrating uniform design and support vector regression is established to replace the time-consuming finite-element model. Su et 
al. [Su, Yu, Xiao et al. (2014)] presented a Gaussian process machine learning method for structural reliability analysis by combining the Gaussian process and importance sampling approach.

\subsection{Construction site management}

Because of the dynamic and complex work conditions on-site, it is a difficult task to ensure personal safety in construction. Currently, the computer vision techniques for target recognition that monitor the locations of workers may require longer computational cycles. Against this backdrop, Ding et al. [Ding, Fang, Luo et al. (2018)] developed a hybrid deep learning model that integrates convolutional neural network (CNN) and long short-term memory (LSTM) to automatically identify unsafe behaviors of employees on construction sites. The CNN model is applied to each frame to capture the spatial features obtained from video, and LSTM network is used to understand the temporal information from the continuous frames that are generated.

In the data from ground penetrating radar (GPR) exploration of concrete bridge decks, it is a time-consuming and laborious work to manually pick rebars. Dinh et al. [Dinh, Gucunski and Duong (2018)] proposed an automated rebar localization and detection algorithm, which combined image processing technology with deep convolutional neural networks (CNNs). Firstly, the image processing methods are used to locate the pixels containing the potential rebar peak. Then, the windowed images around the potential pixel are extracted from the GPR scans. Finally, those are classified by a trained CNN.

There are many visual monitoring methods for construction site, but current technology has not reached the accuracy required for robust identification of construction equipment in the actual construction site images. To solve this problem, Kim et al. [Kim, Hong and Byun (2018)] proposed a construction equipment detection model based on deep convolutional network (Fig. 11), which is helpful for construction site management. This model is trained with a small amount of construction equipment data through transfer learning.

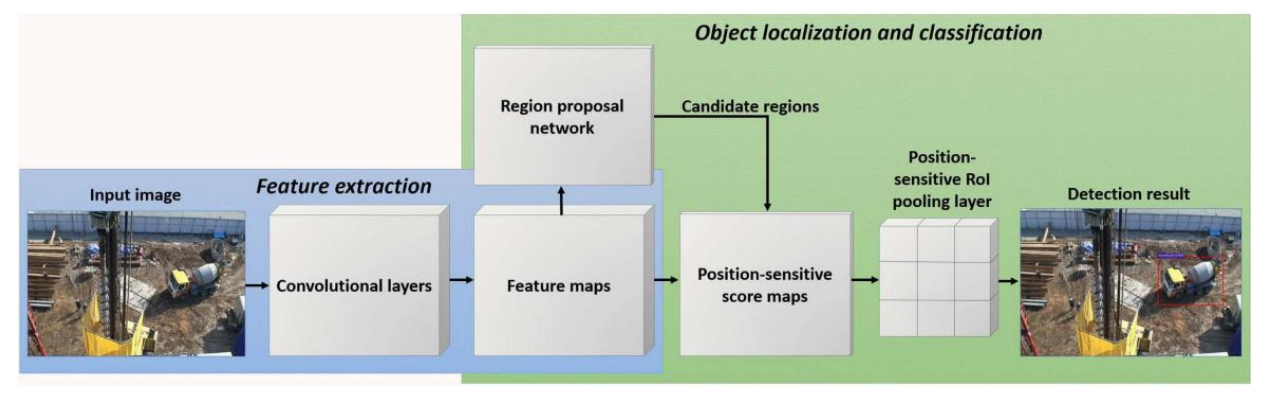

Figure 11: R-FCN-based construction equipment detection pipeline [Kim, Hong and Byun (2018)]

The effective performance of the guardrail system can ensure the safety of construction officers on site, but not all the missing safety guardrail situation can be found in time due to the limited-number safety officers. Kolar et al. [Kolar, Chen and Luo (2018)] presented a safety guardrail detection model based on convolutional neural network (CNN). The background image was added to the three- dimensional model of guardrail, which 
generated an augmented data set, used as the training set. Transfer learning is utilized and the basic features extraction for the neural network was constructed by using the Visual Geometry Group architecture with 16 layers (VGG-16) model.

\subsection{Other applications}

Much research has been carried out on the prediction of pavement friction using pavement texture information. However, the models based on the traditional pavement texture index are not enough to predict the friction performance of the assorted pavement surfaces under different influencing conditions. Yang et al. [Yang, Li, Zhan et al. (2018)] developed a convolutional neural network $(\mathrm{CNN})$ based deep learning architecture, FrictionNet, which can directly predict the level of pavement friction by using the texture profiles. This architecture consists of six artificial neuron layers: two convolution layers, three fully connected layers and one output layer, with 606,409 tuned hyper-parameters.

The fluctuation of economic variables and indicators such as liquidity, wholesale price index and construction service index will lead to variation in costs, which is usually ignored in the traditional cost estimation calculation. To solve this problem, considering economic variables and indicators, Rafiei et al. [Rafiei and Adeli (2018b)] developed an innovative construction cost estimation model, which includes an unsupervised deep Boltzmann machine (DBM) learning method along with a softmax layer, as well as a three-layer back-propagation neural network (BPNN).

At present, most studies use convolutional neural networks (CNNs) as feature extractor for classification/recognition, but less to perform regression or function approximation problems. As the first attempt, $\mathrm{Wu}$ et al. [Wu and Jahanshahi (2019)] tried to use deep $\mathrm{CNN}$ for response estimation in structural dynamic problems. The vibration responses of a linear single-degree- of-freedom (SDOF) system, a nonlinear SDOF system, and a fullscale 3-story multidegree of freedom (MDOF) steel frame are estimated by a deep CNNbased approach.

Moreover, since determining the shear strength of soil is very important for construction project, Bui et al. [Bui, Hoang and Nhu (2019)] proposed a swarm intelligence-based machine learning approach for predicting soil shear strength for road construction, which is a hybrid AI model that integrates the least squares support vector machine (LSSVM) and the cuckoo search optimization. Pham et al. [Pham, Son, Hoang et al. (2018)] also predict the shear strength of soft soil using four machine learning methods: particle swarm optimization-adaptive network based fuzzy inference system (PANFIS), genetic algorithm-ANFIS, support vector regression, and ANN.

In the context of big data, only relatively complex models, or models with strong expressive ability, can fully explore the abundant information contained in large-scale data. The deep learning method can make use of a large amount of data, which means it can constantly improve itself with more data [Lin, Nie and Ma (2017)]. As a result, they have become key approaches to the concept of "big data". With more powerful depth models, we may be able to extract more valuable information and knowledge from big data. So, in the next few years, we will see more and more examples of depth models applied to big data in engineering. 


\section{Conclusion}

This paper reviews the application and development of artificial intelligence in civil engineering over the years. Artificial intelligence algorithm and neural network have been applied in the field of civil engineering for decades, widely used in structural optimization, structural state assessment and health monitoring, construction engineering, bridge engineering, geotechnical engineering, highway engineering and so on.

In recent years, Big Data technologies and deep learning have been successfully applied in various aspects of civil engineering, among which the Big Data technologies have developed fastest in structural maintenance. With the rapid development of computer vision based on deep learning, the structure health monitoring based on computer vision has been greatly improved. However, deep learning has made few breakthroughs in other aspects of civil engineering. In the era of big data, deep learning becomes an efficient tool, which can fully tap into the rich information contained in big data. Therefore, the combination of big data and deep learning will become a new research direction of artificial intelligence in civil engineering.

Acknowledgement: This work has been supported by the Chinese National Natural Science Foundation (51208126, 51578169) and Guangzhou Municipal Science and Technology Bureau in China (201904010307).

\section{References}

Abdelaty, A.; Jeong, H. D.; Smadi, O. (2018): Barriers to implementing data-driven pavement treatment performance evaluation process. Journal of Transportation Engineering Part B-Pavements, vol. 144, no. 4.

Adeli, H. (2001): Neural networks in civil engineering: 1989-2000. Computer-Aided Civil and Infrastructure Engineering, vol. 16, no. 2, pp. 126-142.

Adeli, H.; Yeh, C. (1989): Preceptron learning in engineering design. Microcomputer in Civil Engineering, vol. 4, pp. 247-256.

Artar, M.; Daloglu, A. T. (2018): Optimum weight design of steel space frames with semi-rigid connections using harmony search and genetic algorithms. Neural Computing \& Applications, vol. 29, no. 11, pp. 1089-1100.

Aziz, Z.; Riaz, Z.; Arslan, M. (2017): Leveraging BIM and big data to deliver well maintained highways. Journal of Facilities, vol. 35, no. 13-14, pp. 818-832.

Bao, Y. Q.; Tang, Z. Y.; Li, H.; Zhang, Y. F. (2019): Computer vision and deep learning-based data anomaly detection method for structural health monitoring. Structural Health Monitoring-An International Journal, vol. 18, no. 2, pp. 401-421.

Barr, A.; Feigenbaum, E. A. (1981): The Handbook of Artificial Intelligence. Morgan Kaufmann, USA.

Basheer, I. A.; Hajmeer, M. (2000): Artificial neural networks: fundamentals, computing, design, and application. Journal of Microbiological Methods, vol. 43, no. 1, pp. 3-31. 
Bilal, M.; Oyedele, L. O.; Akinade, O. O.; Ajayi, S. O. (2016): Big data architecture for construction waste analytics (CWA): a conceptual framework. Journal of Building Engineering, vol. 6, pp. 144-156.

Bonabeau, E.; Dorigo, M.; Theraulaz, G. (1999): Swarm Intelligence: From Natural to Artificial Systems. Oxford University Press, USA.

Bui, D. T.; Hoang, N.; Nhu, V. (2019): A swarm intelligence-based machine learning approach for predicting soil shear strength for road construction: a case study at Trung Luong National Expressway Project (Vietnam). Engineering with Computers, vol. 35, no. 3, pp. 955-965.

Cai, G. W.; Mahadevan, S. (2018): Big data analytics in uncertainty quantification: application to structural diagnosis and prognosis. ASCE-ASME Journal of Risk and Uncertainty in Engineering Systems Part A-Civil Engineering, vol. 4, no. 1.

Cao, C. Z. (2010): Artificial Intelligence Technology. Tsinghua University Press, China.

Cascardi, A.; Micelli, F.; Aiello, M. A. (2017): An artificial neural networks model for the prediction of the compressive strength of FRP-confined concrete circular columns. Engineering Structures, vol. 140, pp. 199-208.

Catbas, F. N.; Malekzadeh, M. (2016): A machine learning-based algorithm for processing massive data collected from the mechanical components of movable bridges. Automation in Construction, vol. 72, pp. 269-278.

Cha, Y. J.; Choi, W.; Buyukozturk, O. (2017): Deep learning-based crack damage detection using convolutional neural networks. Computer-Aided Civil and Infrastructure Engineering, vol. 32, no. 5, pp. 361-378.

Cha, Y. J; Choi, W.; Suh, G.; Mahmoudkhani, S.; Buyukozturk, O. (2018): Autonomous structural visual inspection using region-based deep learning for detecting multiple damage types. Computer-Aided Civil and Infrastructure Engineering, vol. 33, pp. 731-747.

Chatterjee, S.; Sarkar, S.; Hore, S.; Dey, N.; Ashour, A. S. et al. (2017): Particle swarm optimization trained neural network for structural failure prediction of multistoried RC buildings. Neural Computing \& Applications, vol. 28, pp. 2005-2016.

Chen, G. R.; Li, T. G.; Chen, Q. J.; Ren, S. F.; Wang, C. et al. (2019): Application of deep learning neural network to identify collision load conditions based on permanent plastic deformation of shell structures. Computational Mechanics, vol. 64, no. 2, pp. 435-449.

Chen, X. L.; Fu, J. P.; Yao, J. L.; Gan J. F. (2018): Prediction of shear strength for squat RC walls using a hybrid ANN-PSO model. Engineering with Computers, vol. 34, no. 2, pp. 367-383.

Chokor, A.; ElAsmar, M. (2017): Data-driven approach to investigate the energy consumption of LEED-certified research buildings in climate zone 2B. Energy Engineering, vol. 143 , no. 2.

Chou, J. S.; Ngo, N. T. (2016): Smart grid data analytics framework for increasing energy savings in residential buildings. Automation in Construction, vol. 72, pp. 247-257. 
Colorni, M.; Dorigo, M.; Maniezzo, V. (1992): Distributed optimization by ant colonies. Proceedings of the First European Conference on Artificial Life, pp. 134-142.

Delgado, J. M. D.; Butler, L. J.; Brilakis, I.; Elshafie, M. Z. E. B.; Middleton, C. R. (2018): Structural performance monitoring using a dynamic data-driven BIM environment. Journal of Computing in Engineering, vol. 32, no. 3.

Deng, L.; Yu, D. (2014): Deep learning: methods and applications. Foundations and Trends in Signal Processing, vol. 7, no. 3-4, pp. 1-199.

Ding, L. Y.; Fang, W. L.; Luo, H. B.; Love, P. E. D.; Zhong, B. T. et al. (2018): A deep hybrid learning model to detect unsafe behavior: integrating convolution neural networks and long short-term memory. Automation in Construction, vol. 86, pp. 118-124.

Dinh, K.; Gucunski, N.; Duong, T. H. (2018): An algorithm for automatic localization and detection of rebars from GPR data of concrete bridge decks. Automation in Construction, vol. 89, pp. 292-298.

Dorigo, M. (1992): Optimization, Learning and Natural Algorithm (Ph.D. Thesis). University of Politecnico di Milano, Italy.

Dorigo, M.; Caro, G. D. (1999): The ant colony optimization meta-heuristic. New Ideas in Optimization, pp. 11-32.

Dorigo, M.; Caro, G. D.; Gambardella, L. M. (1999): Ant algorithms for discrete optimization. Artificial Life, vol. 5, no. 2, pp. 137-172.

Dorigo, M.; Maniezzo, V.; Colorni, A. (1991): Positive feedback as a search strategy. Technical Report, pp. 91-106.

Dung, C. V.; Anh, L. D. (2019): Autonomous concrete crack detection using deep fully convolutional neural network. Automation in Construction, vol. 99, pp. 52-58.

Gandomi, A. H.; Alavi, A. H. (2012): Krill herd: a new bio-inspired optimization algorithm. Communications in Nonlinear Science and Numerical Simulation, vol. 12, no. 12, pp. 4831-4845.

Gandomi, A. H.; Yang, X. S.; Talatahari, S.; Alavi, A. H. (2013): Firefly algorithm with chaos. Communications in Nonlinear Science and Numerical Simulation, vol. 18, no. 1, pp. 89-98.

Gandomi, A. H.; Alavi, A. H. (2016): An introduction of krill herd algorithm for engineering optimization. Journal of Civil Engineering and Management, vol. 22, no. 3, pp. 302-310.

Gandomi, A. H.; Sajedi, S.; Kiani, B.; Huang, Q. D. (2016): Genetic programming for experimental big data mining: a case study on concrete creep formulation. Automation in Construction, vol. 70, pp. 89-97.

Gan, M.; Wang, C.; Zhu, C. A. (2016): Construction of hierarchical diagnosis network based on deep learning and its application in the fault pattern recognition of rolling element bearings. Mechanical Systems and Signal Processing, vol. 72-73, pp. 92-104.

Gao, W. (2016): Determination of the noncircular critical slip surface in slope stability analysis by meeting ant colony optimization. Journal of Computing in Civil Engineering, vol. 30 , no. 2 . 
Gao, Y. Q.; Mosalam, K. M. (2018): Deep transfer learning for image-based structural damage recognition. Computer-Aided Civil and Infrastructure Engineering, vol. 33, no. 9, pp. 748-768.

Garcia-Segura, T.; Yepes, V.; Frangopol, D. M. (2017): Multi-objective design of post-tensioned concrete road bridges using artificial neural networks. Structural and Multidisciplinary Optimization, vol. 56, no. 1, pp. 139-150.

Goldberg, D. E. (1989): Genetic Algorithms in Search, Optimization and Machine Learning. Longman Press, USA.

Gopalakrishnan, K.; Khaitan, S. K.; Choudhary, A.; Agrawal A. (2017): Deep convolutional neural networks with transfer learning for computer vision-based datadriven pavement distress detection. Construction and Building Materials, vol. 157, pp. 322-330.

Gui, G. Q.; Pan, H.; Lin, Z. B.; Li, Y. H.; Yuan, Z. J. (2017): Data-driven support vector machine with optimization techniques for structural health monitoring and damage detection. KSCE Journal of Civil Engineering, vol. 21, no. 2, pp. 523-534.

Guo, L. H.; Wang, G. G.; Gandomi, A. H.; Alavi, A. H.; Duan, H. (2014): A new improved krill herd algorithm for global numerical optimization. Neurocomputing, vol. 138, pp. 392-402.

Hackwood, S.; Beni, G. (1922): Self-organization of sensors for swarm intelligence. IEEE International Conference on Robotics and Automation, vol. 1, pp. 819-829.

Han, K. K.; Golparvar-Fard, M. (2017): Potential of big visual data and building information modeling for construction performance analytics: an exploratory study. Automation in Construction, vol. 73, pp. 184-198.

Hasanipanah, M.; Noorian-Bidgoli, M.; Armaghani D. J.; Khamesi H. (2016): Feasibility of PSO-ANN model for predicting surface settlement caused by tunneling. Engineering with Computers, vol. 32, no. 4, pp. 705-715.

Hecht-Nielsen, R. (1990): Neurocomputing. Addison-Wesley Press, UK.

Hinton, G. E.; Salakhutdinov, R. R. (2006): Reducing the dimensionality of data with neural networks. Science, vol. 313, pp. 504-507.

Holland, J. H. (1975): Adaptation in Natural and Artificial Systems. University of Michigan Press, USA.

Huang, H. W.; Li, Q. T.; Zhang, D. M. (2018): Deep learning based image recognition for crack and leakage defects of metro shield tunnel. Tunnelling and Underground Space Technology, vol. 77, pp. 166-176.

Jamshidi, A.; Hahizadeh, S.; Su, Z.; Naeimi, M.; Nunez, A. et al. (2018): A decision support approach for condition-based maintenance of rails based on big data analysis. Transportation Research Part C-Emerging Technologies, vol. 95, pp. 185-206.

Jong, K. A.; Dejong, K. (1975): The Analysis of the Behavior of a Class of Genetic Adaptive Systems. The University of Michigan Press, USA.

Kalogirou, S. A. (2001): Artificial neural networks in renewable energy systems applications: a review. Renewable \& Sustainable Energy Reviews, vol. 5, no. 4, pp. 373-401. 
Kalogirou, S. A. (2003): Artificial intelligence for the modeling and control of combustion processes: a review. Progress in Energy and Combustion Science, vol. 29, no. 6, pp. 515-556.

Kang, F.; Li, J. J. (2016): Artificial bee colony algorithm optimized support vector regression for system reliability analysis of slopes. Journal of Computing in Civil Engineering, vol. 30, no. 3, 04015040.

Karaboga, D. (2005): An Idea Based on Honey Bee Swarm for Numerical Optimization. Erciyes University, Turkey.

Kennedy, J.; Eberhart, R. (1995): Particle swarm optimization. Proceedings of the 1995 IEEE International Conference on Neural Networks, vol. 4, pp. 1942-1948.

Kim, H.; Kim, H.; Hong, Y. W.; Byun, H. (2018): Detecting construction equipment using a region- based fully convolutional network and transfer learning. Journal of Computing in Civil Engineering, vol. 32, no. 2, 04017082.

Kim, Y. J.; Queiroz, L. B. (2017): Big data for condition evaluation of constructed bridges. Engineering Structures, vol. 141, pp. 217-227.

Kolar, Z.; Chen, H. N.; Luo, X. W. (2018): Transfer learning and deep convolutional neural networks for safety guardrail detection in 2D images. Automation in Construction, vol. 89, pp. 58-70.

Konak, A.; Coit, D. W.; Smith, A. E. (2005): Multi-objective optimization using genetic algorithms: a tutorial. Reliability Engineering \& System Safety, vol. 91, no. 9, pp. 992-1007.

Konar, A. (1999): Artificial Intelligence and Soft Computing Behavioral and Cognitive Modeling of the Human Brain. CRC Press, USA.

Kubair, K. S.; Mohan, S. C. (2018): Numerical model updating technique for structures using firefly algorithm. International Conference on Recent Advanced in Materials, Mechanical and Civil Engineering, vol. 330, UNSP012123.

LeCun, Y.; Bengio, Y.; Hinton, G. (2015): Deep learning. Nature, vol. 521, pp. 436-444. Li, R. X.; Yuan, Y. C.; Zhang, W.; Yuan, Y. L. (2018): Unified vision-based methodology for simultaneous concrete defect detection and geolocalization. ComputerAided Civil and Infrastructure Engineering, vol. 33, no. 7, pp. 527-544.

Liang, Y.; Wu, D. L.; Liu, G. R.; Li, Y. H. (2016): Big data-enabled multiscale serviceability analysis for aging bridges. Digital Communications and Networks, vol. 2, no. 3, pp. 97-107.

Lieu, Q. X.; Do, D. T. T.; Lee, J. (2018): An adaptive hybrid evolutionary firefly algorithm for shape and size optimization of truss structures with frequency constraints. Computers \& Structures, vol. 195, pp. 99-112.

Lin, Y. Z.; Nie, Z. H.; Ma, H. W. (2017): Structural damage detection with automatic feature-extraction through deep learning. Computer-Aided Civil and Infrastructure Engineering, vol. 32, no. 12, pp. 1025-1046.

Liu, L. L.; Yan, R. J.; Maruvanchery, V.; Kayacan, E.; Chen, I. M. et al. (2017): Transfer learning on convolutional activation feature as applied to a building quality 
assessment robot. International Journal of Advanced Robotic Systems, vol. 14, no. 3, pp. $1-12$.

Lu, N. W.; Noori, M.; Liu, Y. (2017): Fatigue reliability assessment of welded steel bridge decks under stochastic truck loads via machine learning. Journal of Bridge Engineering, vol. 22, no. 1, 04016105.

Lu, P. Z.; Chen, S. Y.; Zheng, Y. J. (2012): Artificial intelligence in civil engineering. Mathematical Problems in Engineering.

Matarazzo, T. J.; Santi, P.; Pakzad, S. N.; Carter, K.; Ratti, C. et al. (2018): Crowdsensing framework for monitoring bridge vibrations using moving smartphones. Proceedings of IEEE, vol. 106, no. 4, pp. 577-593.

McCulloch, W.; Pitts, W. (1943): A logical calculus of the ideas immanent in nervous activity. Bulletin of Mathematical Biophysics, vol. 52, no. 1-2, pp. 99-115.

Mehrkian, B.; Bahar, A.; Chaibakhsh, A. (2019): Semiactive conceptual fuzzy control of magnetorheological dampers in an irregular base-isolated benchmark building optimized by multi- objective genetic algorithm. Structural Control \& Health Monitoring, vol. 26 , pp. 1-28.

Meijer, E. (2011): The world according to LINQ. Communications of the ACM, vol. 54, no. 11, pp. 45-51.

Mellit, A.; Kalogirou, S. A. (2008): Artificial intelligence techniques for photovoltaic applications: a review. Progress in Energy and Combustion Science, vol. 34, no. 5, pp. 574-632.

Motawa, I. (2017): Spoken dialogue BIM systems-an application of big data in construction. Journal of Facilities, vol. 35, no. 13, pp. 787-800.

Mroginski, J. L.; Beneyto, P. A.; Gutierrez, G. J.; Di, R. A. (2016): A selective genetic algorithm for multiobjective optimization of cross sections in $3 \mathrm{D}$ trussed structures based on a spatial sensitivity analysis. Multidiscipline Modeling in Materials and Structures, vol. 12, no. 2, pp. 423-435.

Naderpour, H.; Rafiean, A. H.; Fakaharian, P. (2018): Compressive strength prediction of environmentally friendly concrete using artificial neural networks. Journal of Building Engineering, vol. 16, pp. 213-219.

Ni, F. T.; Zhang, J.; Chen, Z. Q. (2019): Pixel-level crack delineation in images with convolutional feature fusion. Structural Control and Health Monitoring, vol. 26, no. 1, e2286.

Pan, H.; Azimi, M.; Yan, F.; Lin, Z. B. (2018): Time-frequency-based data-driven structural diagnosis and damage detection for cable-stayed bridges. Journal of Bridge Engineering, vol. 23, no. 6, 04018033.

Pathirage, C. S. N.; Li, J.; Li, L.; Hao, H.; Liu, W. Q. et al. (2018): Structural damage identification based on autoencoder neural networks and deep learning. Engineering Structures, vol. 172, pp. 13-28.

Pham, B. T.; Son, L. H.; Hoang, T. A.; Nguyen, D. M.; Bui, D. T. (2018): Prediction of shear strength of soft soil using machine learning methods. Catena, vol. 166, pp. 181-191. 
Qin, S. Q.; Zhang, Y. Z.; Zhou, Y. L.; Kang, J. T. (2018): Dynamic model updating for bridge structures using the kriging model and PSO algorithm ensemble with higher vibration modes. Sensors, vol. 18, no. 6, pp. 1879.

Rafiei, M. H.; Adeli, H. (2018a): A novel unsupervised deep learning model for global and local health condition assessment of structures. Engineering Structures, vol. 156, pp. 598-607.

Rafiei, M. H.; Adeli, H. (2018b): Novel machine-learning model for estimating construction costs considering economic variables and indexes. Journal of Construction Engineering and Management, vol. 144, no. 12, 04018106.

Rakhshani, H.; Rahati, A. (2017): Intelligent multiple search strategy cuckoo algorithm for numerical and engineering optimization problems. Arabian Journal for Science and Engineering, vol. 42, no. 2, pp. 567-593.

Ren, S. F.; Chen, G. R.; Li, T.; Chen, Q. J.; Li, S. F. (2018): A deep learning-based computational algorithm for identifying damage load condition: an artificial intelligence inverse problem solution for failure analysis. Computer Modeling in Engineering \& Sciences, vol. 117, no. 3, pp. 287-307.

Rich, E.; Knight, K. (1996): Artificial Intelligence. McGraw-Hill, USA.

Rosales, M. J.; Liyanapathirana, R. (2017): Data driven innovations in structural health monitoring. 12th International Conference on Damage Assessment of Structures, Japan.

Saleem, M.; DiCaro, G. A.; Farooq, M. (2011): Swarm intelligence based routing protocol for wireless sensor networks: survey and future directions. Information Sciences, vol. 181, no. 20, pp. 4597-4624.

Schalkoff, R. J. (1997): Artificial Neural Networks. McGraw-Hill Press, USA.

Schmidhuber, J. (2015): Deep learning in neural networks: an overview. Neural Networks, vol. 61, pp. 85-117.

Silva, M.; Santos, A.; Figueiredo, E.; Santos, R.; Sales, C. et al. (2016): A novel unsupervised approach based on a genetic algorithm for structural damage detection in bridges. Engineering Applications of Artificial Intelligence, vol. 52, pp. 168-180.

Su, G. S.; Yu, B.; Xiao, Y. L.; Yan, L. B. (2014): Gaussian process machine-learning method for structural reliability analysis. Advances in Structural Engineering, vol. 17, no. 9, pp. 1257-1270.

Tran, C. (2016): Structural-damage detection with big data using parallel computing based on MPSOC. International Journal of Machine Learning and Cybernetics, vol. 7, no. 6, pp. 1213-1223.

Truong, V. H.; Nguyen, P. C.; Kim, S. E. (2017): An efficient method for optimizing space steel frames with semi-rigid joints using practical advanced analysis and the microgenetic algorithm. Journal of Construction Steel Research, vol. 128, pp. 416-427.

Tseranidis, S.; Brown, N. C.; Mueller, C. T. (2016): Data-driven approximation algorithms for rapid performance evaluation and optimization of civil structures. Automation in Construction, vol. 72, pp. 279-293. 
Walker, S. (2014): Big data: a revolution that will transform how we live, work, and think. International Journal of Advertising, vol. 33, no. 1, pp. 181-183.

Wang, C.; Abdul-Rahman, H.; Chow, P. S. (2016): Development and test run of civil engineering schedule acceleration model through ant colony optimization. Journal of Civil Engineering and Management, vol. 22, no. 8, pp. 1009-1020.

Wang, G. G.; Gandomi, A. H.; Alavi, A. H. (2014): Stud kill herd algorithm. Neurocomputing, vol. 128, pp. 363-370.

Wang, G. G.; Guo, L. H.; Gandomi, A. H.; Hao, G. S.; Wang, H. Q. (2014): Chaotic krill herd algorithm. Information Sciences, vol. 274, pp. 17-34.

Wu, R. T.; Jahanshahi, M. R. (2019): Deep convolutional neural network for structural dynamic response estimation and system identification. Journal of Engineering Mechanics, vol. 145, no. 1, 04018125.

Wu, X. D.; Zhu, X. Q.; Wu, G. W.; Ding, W. (2014): Data mining with big data. IEEE Transactions on Knowledge and Data Engineering, vol. 26, no. 1, pp. 97-107.

Xiao, X. L.; Zhou, C. R. (2017): Application and challenges of big data in quality monitoring of highway engineering. International Conference on Advances in Materials, China.

Xu, Y.; Li, S. L.; Zhang, D. Y.; Jin, Y.; Zhang, F. J. et al. (2018): Identification framework for cracks on a steel structure surface by a restricted Boltzmann machines algorithm based on consumer-grade camera images. Structural Control and Health Monitoring, vol. 25, no. 2, e2075.

Xu, Y.; Wei, S. Y.; Bao, Y. Q.; Li, H (2019): Automatic seismic damage identification of reinforced concrete columns from images by a region-based deep convolutional neural network. Structural Control and Health Monitoring, vol. 26, no. 3, e2313.

Xue, X. H.; Xiao, M. (2017): Deformation evaluation on surrounding rocks of underground caverns based on PSO-LSSVM. Tunnelling and Underground Space Technology, vol. 69, pp. 171-181.

Yan, F.; Lin, Z. B.; Azarmi, F.; Sobolev, K. (2017): Evaluation and prediction of bond strength of GFRP-bar reinforced concrete using artificial neural network optimized with genetic algorithm. Composite Structures, vol. 161, pp. 441-452.

Yang, G. W.; Li, Q. J.; Zhan, Y.; Fei, Y.; Zhang, A. N. (2018): Convolutional neural network-based friction model using pavement texture data. Journal of Computing in Civil Engineering, vol. 32, no. 6, 04018052.

Yang, X. S. (2008): Nature-Inspired Metaheuristic Algorithms. Luniver Press, UK.

Yang, X. S.; Deb, S. (2009): Cuckoo search via Lévy flights. Proceedings of World Congress on Nature and Biologically Inspired Computing, pp. 210-214, USA.

Yang, X. S.; Deb, S. (2010): Engineering optimization by cuckoo search. International Journal of Mathematical and Numerical Optimisation, vol. 4, pp. 330-343.

Yang, X. S.; Deb, S. (2013): Multiobjective cuckoo search for design optimization. Computers \& Operations Research, vol. 40, no. 6, pp. 1616-1624. 
Zamani, A. A.; Tavakoli, S.; Etedali, S. (2017): Fractional order PID control design for semi-active control of smart base isolated structures: a multi-objective cuckoo search approach. ISA Transactions, vol. 67, pp. 222-232.

Zarbaf, S. E. H. A. M.; Norouzi, M.; Allemang, R. J.; Hunt, V. J.; Helmicki, A. (2017): Stay cable tension estimation of cable-stayed bridges using genetic algorithm and particle swarm optimization. Journal of Bridge Engineering, vol. 22, no. 10, pp. 1-8.

Zhang, A.; Wang, K. C. P.; Fei, Y.; Liu, Y.; Tao, S. Y. (2018): Deep learning-based fully automated pavement crack detection on $3 \mathrm{~d}$ asphalt surfaces with an improved CrackNet. Journal of Computing in Civil Engineering, vol. 32, no. 5, 04018041.

Zhang, A.; Wang, K. C. P.; Li, B. X.; Yang, E. H.; Dai, X. X. et al. (2017): Automated pixel-level pavement crack detection on 3D asphalt surfaces using a deep-learning network. Computer-Aided Civil and Infrastructure Engineering, vol. 32, no. 10, pp. 805-819.

Zhang, K. G.; Cheng, H. D.; Zhang, B. Y. (2018): Unified approach to pavement crack and sealed crack detection using preclassification based on transfer learning. Journal of Computing in Civil Engineering, vol. 32, no. 2, 04018001.

Zhang, W. G.; Goh, A. T. C.; Zhang, Y. M. (2016): Multivariate adaptive regression splines application for multivariate geotechnical problems with big data. Geotechnical and Geological Engineering, vol. 34, no. 1, pp. 193-204.

Zhao, H. B.; Yin, S. D. (2016): Inverse analysis of geomechanical parameters by the artificial bee colony algorithm and multi-output support vector machine. Inverse Problems in Science and Engineering, vol. 24, no. 7, pp. 1266-1281.

Zhuang, L.; Wang, L.; Zhang, Z. J.; Tsui, K. L. (2018): Automated vision inspection of rail surface cracks: a double-layer data-driven framework. Transportation Research Part C-Emerging Technologies, vol. 92, pp. 258-277.

Zikopoulos, P.; Eaton, C. (2011): Understanding Big Data: Analytics for Enterprise Class Hadoop and Streaming Data. McGraw-Hill Osborne Media, USA. 